\title{
A CHARACTERIZATION OF POINT SEMIUNIFORMITIES
}

\author{
JENNIFER P. MONTGOMERY \\ Lousiana State University at Alexandria \\ Division of Sciences \\ 8100 Highway 71 South \\ Alexandria, Louisiana 71302-9633 \\ (Recelved October 21, 1994)
}

\begin{abstract}
The concept of a uniformity was developed by $A$. Weil and there have been several generalizations. This paper defines a point semiuniformity and gives necessary and sufficient conditions for a topological space to be point semiuniformizable. In addition, just as uniformities are associated with topological groups, a point semiuniformity is naturally associated with a semicontinuous group. This paper shows that a point semiuniformity associated with a semicontinuous group is a uniformity if and only if the group is a topological group.
\end{abstract}

KEY WORDS AND PHRASES: Uniformity, point semiuniformity, vicinities, point semiuniformizable, homogeneous, topological group, semicontinuous group, semifundamental system, point regular, bihomogeneous.

1991 AMS MATHEMATICS SUBJECT CLASSIFICATION CODES: Primary 53E15; Secondary 54 H11.

1. Introduction. In 1937, A. Weil [1] generalized the concept of a metric space by defining a topology-generating structure called a uniformity. There have been several generalizations of uniformities. For example, a semiuniformity, $\mathbb{U}$, for a set $\not{Z}$ is a filter of supersets of the diagonal in $\mathbb{X} \times \mathbb{K}$ such that for each $U$ in $U$, there is a $V$ in $U$ such that $\mathrm{V}^{-1}=\{(y, x) \mid(x, y) \in V\} \subseteq U$. As with a uniformity and its other generalizations, there is a natural way to try to construct neighborhoods of points. Namely, for each $x$ in $X$ and $U$ in $U$ we define a slice, $U[x]$, to be $\{y \mid(x, y) \in U\}$. For a semiuniformity, the collection $\{U[x]\}$ does generate a topology on $x$ but we are left with the unsatisfactory situation that some of the slices are not neighborhoods in this topology. In [2], w. Page gets around this problem by calling a semiuniformity a $t$-semiuniformity (for topological semiuniformity) if all the slices turn out to be neighborhoods, and he proves that a space is t.semiuniformizable (there is a $t$-semiuniformity which generates the topology) if and only if the space satisfies a certain separation property. We take a different approach. We define a point semiuniformity, $\mathbb{P}$, to be a semiuniformity with the added condition that for every $S \in \mathbb{P}$ there is a $T \in \mathbb{P}$ having for each $(x, y) \in T$ a $V \in \mathbb{P}$ such that $(x, y) \circ V$ and $V \circ(x, y)$ are contained in $S$. We will show that the slices gotten from $\mathbb{P}$ will always be neighborhoods in the topology generated by $\mathbb{P}$ and that a space is point semiuniformizable if and only if it satisfies the same separation property referred to above. 
The natural association of uniformities with topological groups, or more exactly, with a fundamental system of a topological group is well known. We show that a point semiuniformity is just as naturally associated with a semicontinuous group [3] (called semitopological groups by Bourbaki [4] and L. Fuchs [5]). A semicontinuous group is a group with a topology making inversion and left and right multiplication by single elements continuous. We show that the point semiuniformity associated with a semicontinuous group is a uniformity if and only if the group is a topological group.

\section{Point Semiuniformity}

We begin by formalizing our definitions. A point semiuniformity for a set $x$ is a filter $\mathbb{P}$ of subsets of $\not \times x$ such that $\forall S \in \mathbb{P}$,
1) $\Delta \subseteq S$
2) $\exists T \in \mathbb{P}$ such that $\mathrm{T}^{-1} \subseteq \mathrm{S}$
3) $\exists T \in \mathbb{P}$ such that for each $(x, y) \in T$ there is a $V \in \mathbb{P}$ with $\mathrm{V} \circ(\mathrm{x}, \mathrm{y}) \subseteq \mathrm{S}$ and $(\mathrm{x}, \mathrm{y}) \circ \mathrm{V} \subseteq \mathrm{S}$.

$A$ pair $(K, \mathbb{P})$ consisting of a set $X$ and a point semiuniformity $\mathbb{P}$ on $X \times \dot{X}$ is called a point semiuniform space. We call $\beta$ a base for a point semiuniformity $\mathbb{P}$ if and only if the collection of all supersets of elements of $\beta$ is $\mathbb{P}$. It is clear that any filter base satisfying the three conditions above is a base for a point semiuniformity.

THEOREM 1. Let $\mathbb{P}$ be a point semiuniformity for a set $X$ and let $\mathbb{P}_{\mathbf{x}}=\{S[\mathbf{X}] \quad \mid \quad S \in \mathbb{P}\}$. Then $\left\{\mathbb{P}_{\mathbf{x}} \mid \mathbf{x} \in \mathbb{K}\right\}$ forms a neighborhood base for a topology $\tau$ on $x$.

PROoF. Clearly, for all $S, T \in \mathbb{P}, \quad x \in S[X]$ and $S[x] \cap T[x]=(S \cap T)[x]$. Now let $S[x] \in \mathbb{P}_{x}$. Since $S \in \mathbb{P}$ then $\exists T \in \mathbb{P}$ with the property that for each $(a, b) \in T, \quad \exists U_{(a, b)} \in \mathbb{P}$ with $U_{(a, b)} \circ(a, b) \leq S$. In addition, since $(x, x) \in T$ then $\exists U_{(x, x)} \in \mathbb{P}$ with $U_{(x, x)} \circ(x, x) \leq S$. Since $U_{(x, x)} \in \mathbb{P}$ then $\exists V \in \mathbb{P}$ with the property given any $(s, t) \in V \quad \exists W_{(s, t)} \in \mathbb{P}$ with $W_{(s, t)} \circ(s, t) \subseteq U_{(x, x)}$. Now $V[x] \in \mathbb{P}_{x}$ and thus we must show that a neighborhood of each point of $V[x]$ is contained in $S[x]$. Suppose that $y \in V[x]$ then $(x, y) \in V$. Now $\exists W_{(x, y)} \in \mathbb{P}$ such that $W_{(x, y)} \circ(x, y) \subseteq U_{(x, x)}$. It suffices to show $W_{(x, y)}[y] \leq S[x]$. Therefore, let $z \in W_{(x, y)}[y]$ and so $(y, z) \in W_{(x, y)}$ which implies that $(x, z) \in W_{(x, y)} \circ(x, y) \subseteq U(x, x)$.

Hence, $(x, z)=(x, z) \circ(x, x) \in U_{(x, x)} \circ(x, x) \leq S$. Consequently, $z \in S[x]$.

A uniformity $\mathbb{U}$ is a semiuniformity with the property that for each $U \in \mathbb{U}$, there is a $V \in \mathbb{U}$ such that $V \_V \subseteq U$. Thus, we see that every uniformity is a point semiuniformity and every $t$-semiuniformity is a point semiuniformity. We now turn our attention to which topologies can be generated by these point semiuniformities. Any topology thus induced is called a point semiuniform topology and the space is called a point semiuniformizable topological space. The finite complement topology on an infinite space is point semiuniformizable, but since the space is not completely regular, it is not uniformizable. 


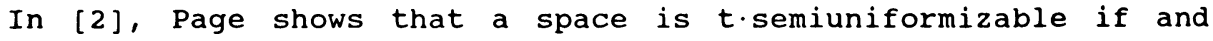
only if $\forall x, y \in \mathbb{R}, \quad x \in C l_{\tau}\{y\}$ iff $y \in C l_{\tau}\{x\}$. We restate this closure condition in an equivalent form.

DEFINITION. A topological space $(x, \tau)$ is point regular (or $p \cdot r e g u l a r)$ if and only if for every $V \in \tau$ and for every $x \in V, C l_{\tau}\{x\} \subseteq V$.

The next proposition states some of the basic topological properties possessed by $\mathrm{p} \cdot$ regular spaces.

PROPOSITION 2 .

i) Every regular space or $\mathrm{T}_{1}$-space is a $\mathrm{p}$-regular space.

ii) A pregular, $\mathrm{T}_{0}$-space is a $\mathrm{T}_{1}$-space.

iii) The continuous closed image of a p.regular space is a p.regular space, but the quotient of a p.regular space need not be p.regular.

iv) Products and subspaces of $p \cdot r e g u l a r$ spaces are p.regular.

v) Although homogeneous spaces need not be p.regular, bihomogeneous spaces are p.regular.

In [2], Page shows that a space $x$ is $t \cdot$ semiuniformizable if and only if it is p.regular. In his proof, he constructs a $t \cdot$ semiuniformity as follows: For each $x \in \mathcal{K}$, let $u_{x}$ be a neighborhood of $x$ and let $R=U\left(x \times u_{x}\right)$ and $S=R \cup R^{-1}$. The collection $\beta$ of all such $S \subseteq X \times X$ forms a base for a $t \cdot s e m i u n i f o r m i t y$ which induces the original topology $\tau$. However, a $t$.semiuniformity need not be a point semiuniformity as the following example shows.

EXAMPLE 3. Consider $\mathbb{R}$, the real numbers, with the usual topology. For each $r \in \mathbb{R}$, choose neighborhoods, $u_{r}$, as follows: Let $u_{1}=\mathbb{R}$. For each element of the sequence $<1-1 / n>\infty, \infty$, choose open interval neighborhoods so that

$$
\begin{gathered}
1 / 2 \notin u_{0}, \quad 0,2 / 3 \notin u_{1 / 2}, \quad 1 / 2,3 / 4 \notin u_{2 / 3} \ldots \\
(k-2) /(k-1), k /(k+1) \notin u_{k-1 / k}, \quad \text { etc. }
\end{gathered}
$$

For $y \in \mathbb{R}-\{1,0,1 / 2,2 / 3,3 / 4 \ldots\}$, choose any neighborhood $u_{y}$ of $y$. Now, let $R=U_{r \in \mathbb{R}}\left(r \times u_{r}\right)$ and let $S=R \cup R^{-1}$. Let $\beta$ be the collection of all such S. Let $B \in \beta$ and we may as well assume $B \subseteq S$. Then there exists $x$ such that $x=1-1 /(m+1), m$ a positive integer, and such that $u_{x}$, the neighborhood of $x$, is strictly contained in $B[1]$, the neighborhood of 1 , since any neighborhood of 1 contains a tail of the sequence $<1-$ $1 / n>\infty, \infty=1$. Then, $(x, 1) \notin x \times u_{x}$ but $(x, 1) \in B[1] \times 1$. In order to have a point semiuniformity, we need $D \circ(x, 1) \subseteq S$ for some $D \in \beta$. The composition

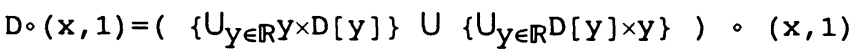

$$
\begin{aligned}
& =(x \times D[1]) \cup\{(x, y) \mid 1 \in D[y]\}
\end{aligned}
$$


and recall that $S=\left(U\left(r \times u_{r}\right)\right) \cup\left(U\left(u_{r} \times r\right)\right)$. Now if $x \times D[1] \subseteq U_{r \in \mathbb{R}} u_{r} \times r$ then $\forall z \in D[1], \quad x \in u_{z}$. But $x=1-1 / m^{\prime} \in D[1]$ for some integer $m^{\prime}>m$ and $x \notin u_{q} \cdot$ Also, $(x, x) \notin x \times u_{x}$. Hence, although $(x, x) \in X \times D[1] \subseteq D \circ(x, 1)$, $(x, x) \notin\left(U_{r \in \mathbb{R}}\left(r \times u_{r}\right)\right) \cup\left(U_{r \in \mathbb{R}}\left(u_{r} \times r\right)\right)$. Thus, $D \circ(x, 1) \notin S$.

This example shows that Page's construction of a $t$-semiuniformity may not be a point semiuniformity. Although $t$.semiuniformities and point semiuniformities are not the same, they are related as the next theorem shows.

THEOREM 4. A topological space $(k, \tau)$ is point semiuniformizable if and only if $(x, \tau)$ is p.regular.

PROOF. Since one direction is trivial, we only need to show that a p.regular space is point semiuniformizable.

Thus, let $\beta$ be the collection of all neighborhoods of $\Delta$ in $\tau \times \tau$. clearly, $\beta$ is a filter that satisfies property 1 ) and 2) of the definition of a point semiuniformity. Therefore, to show property 3 ), let $B \in \beta$ which implies that there exists $U \in \tau \times \tau$ with $\Delta \leq U \leq B$. Let $\mathrm{C}=\mathrm{UnU}^{-1}$. Then $\mathrm{C}$ is open and symmetric and $\Delta \subseteq \mathrm{C} \subseteq \mathrm{B}$. Pick $(x, y) \in C$. We need to find $D \in \beta$ with $D \circ(x, y) \subseteq B$ or equivalently, $D[y] \subseteq C[x] \subseteq B[x]$ (The proof of $(x, y) \circ D \subseteq B$ is similar using inverse notation). Let $D=C-\{y \times$ $(x-c[x])\}$.

a) To show that $D[y] \subseteq C[x]$, suppose that $z \notin C[x]$. Then $(y, z) \in y \times(x-C[x])$ and so $(y, z) \notin D$. Thus, $z \notin D[y]$.

b) To show that $D$ is a neighborhood of $\Delta$, consider $D^{\prime}=C$ $\left\{C l_{\tau}\{y\} \times(x-C[x])\right\}$.

Since $y \times(x-c[x]) \subseteq C l_{\tau}\{y\} \times(x-c[x])$, we have that $D^{\prime} \subseteq D$. Thus, since $D^{\prime}$ is open, we only need to show that $\Delta \subseteq D^{\prime}$.

Case 1] Suppose $(z, z) \in \Delta$ with $z \in C l_{\tau}\{y\}$. Since $(x, \tau)$ is p.regular, $y \in C l_{\tau}\{z\}$ and since $(x, y) \in C$ implies that $y \in C[x]$, then $z \in C[x]$. Thus, $(z, z) \in C-\left\{C l_{\tau}\{y\} \times(x-C[x]) \quad\right\}=D^{\prime}$.

Case 2] Suppose $(z, z) \in \Delta$ with $z \notin C l_{\tau}\{y\}$. Then $(z, z) \in D^{\prime}$ and hence, $\Delta \subseteq D^{\prime}$.

clearly, $B[x] \in \tau, \forall B \in \beta$ and $B \in \tau \times \tau$ which implies that the topology on $x$ generated by $\beta$ is contained in $\tau$. Conversely, let $x \in W \in \tau$. Choose a cover of $X$ by $\tau$-open neighborhoods $\left\{V_{y} \mid y \in X\right\}$ such that $V_{x} \leq W$ and $x \in X-v_{y}$ for $y \notin C l_{\tau}\{x\}$ and $v_{y}=v_{x}$ if $y \in C l_{\tau}\{x\}$ (or equivalently, $x \in C l_{\tau}\{y\}$ ). Let $S=U_{Y \in X} V_{y} \times V_{y}$. clearly, $\Delta s S$ and $S$ is open in $x \times X$. Also, $S[x]=U_{x \in V_{y}} V_{y}=V_{x}$. Hence, $x \in S[x]=V_{x} \leq w$. Therefore, $w$ is open in the topology on $x$ generated by $\beta$. Thus, $\tau$ is contained in the topology on $\mathrm{X}$ generated by $\beta$.

Although we used neighborhoods of $\Delta$ in the product topology in the proof above, we could have used neighborhoods of $\Delta$ in $(\tau \times$ discrete) $\cap$ (discrete $\times \tau$ ) which would give us a finer point semiuniformity inducing the same topology..

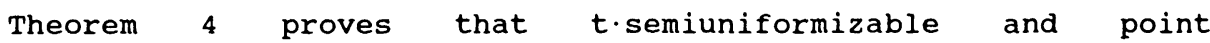
semiuniformizable are equivalent notions for a topological space; 
however, this is their only similarity. From the examples we note that vicinities in a $t$-semiuniform base are constructed simply by forming "crosses" along the diagonal; whereas, in a point semiuniform base vicinities are more carefully constructed possessing "crosses" at each point.

PROPOSITION 5. Let $\left\{U_{\alpha}\right\}_{\alpha \in \Lambda}$ be a collection of point semiuniformities for $x$. Let $\beta$ be the collection of all finite intersections of elements of $U_{\alpha \in \Lambda} U_{\alpha} . \quad \beta$ is a base for a point semiuniformity which is the join of the family $\left\{U_{\alpha}\right\}_{\alpha \in \Lambda}$.

Let $\left\{\mathrm{V}_{\alpha}\right\}_{\alpha \in \Lambda}$ be a family of point semiuniformities for the set $x$. Let $\mathbb{C}$ be the family of all point semiuniformities coarser than each $\mathrm{V}_{\alpha}, \alpha \in \Lambda . \quad \mathbb{C}$ is a nonempty collection since $\{\boldsymbol{K} \times \mathbf{K}\}$ is a point semiuniformity coarser than each $V_{\alpha}, \alpha \in \Lambda$. Then the meet of the family $\left\{\mathrm{v}_{\alpha}\right\}_{\alpha \in \Lambda}$ is $\mathrm{v}_{U \in \mathbb{C}^{U}}$.

Thus, if we let $x$ be a fixed set and we consider the collection of point semiuniformities for $x$, then this collection forms a complete lattice when ordered by set inclusion.

THEOREM 6. If $\beta$ is a finite base for a point semiuniformity then $\beta$ is a base for a uniformity.

PROOF. Let $B \in \beta$. Since $\beta$ is a base for a point semiuniformity, then there exists $C \in \beta$ such that for each $(x, y) \in C$ there exists $D_{(x, y)} \in \beta$ with $D_{(x, y)} \circ(x, y) \subseteq B$. Now, since $\beta$ is a finite base, then the set $S=$ $\left\{D_{(x, y)} \mid(x, y) \in C\right.$ and $\left.D_{(x, y)} \circ(x, y) \subseteq B\right\}$ is finite. Thus, there exists $E \in \beta$ with $E \subseteq(\cap S) \cap C$. Let $(s, t) \in E \circ E$. Then there exists $w$ such that $(s, w) \in E$ and $(w, t) \in E$. Since $E \subseteq(n s) \cap C$, which implies that $(s, w) \in C$, then $(w, t) \in D_{(s, w)}$. Therefore, $(s, t)=(w, t) \circ(s, w) \in D_{(s, w)} \circ(s, w) \subseteq B .4$

COROLLARY 7. On a finite set, point semiuniformities and uniformities coincide.

\section{Semicontinuous Groups}

It is well known that group topologies on a group $G$ are characterized by fundamental systems and fundamental systems give rise to left and right uniformities which give the same topology. A semifundamental system $\mathbf{S}$ for a group $\mathbb{G}$ is a collection of subsets of $\mathbb{G}$ each containing the identity and satisfying the following properties:

1) If $U, V \in S$ then $\exists W \in S$ such that $W \subseteq U n V$

2) If $U \in S$ and $a \in U$ then $\exists V \in S$ such that $V a \leq U$

3) If $U \in \mathbb{S}$ then $\exists V \in S$ such that $V^{-1} \subseteq U$

4) If $U \in \mathbb{S}$ and $x \in \mathbb{G}$ then $\exists V \in \mathbb{S}$ such that $x V x^{-1} \subseteq U$.

E. Clay [3] showed that every semicontinuous group has a semifundamental system. Let $U$ be an element of a semifundamental system for a semicontinuous group. Since inversion is continuous, we 
can always find a symmetric $\mathrm{V}$ such that $\mathrm{V} \subseteq \mathrm{U}$ by letting $\mathrm{V}=\mathrm{UnU}^{-1}$. Consequently, we can always assume that our semifundamental system is a symmetric semifundamental system. The next theorem shows that every semicontinuous group is point semiuniformizable.

THEOREM 8. Let $\mathbf{S}$ be a symmetric semifundamental system for a semicontinuous group $(\mathbb{G}, \tau)$. Define $L_{U}=\{(x, y) \mid x \in Y U\} \quad\left[R_{U}=\{(x, y)\right.$ । $x \in U y$ $\}$ Then $L=\left\{L_{U} \mid U \in S\right\} \quad\left[R=\left\{R_{U} \mid U \in S\right\}\right]$ is a base for a point semiuniformity which induces the original topology $\tau$. The point semiuniformity generated by $L[R]$ is called the left [right] point semiuniformity of $(\mathbb{G}, \tau)$ and is the unique point semiuniformity for $\mathbb{G}$ that generates $\tau$ and has a base of left [right] invariant sets.

PROOF. Clearly, $L$ is a filter base that satisfies the first two properties of a base for a point semiuniformity.

Let $L_{u} \in L$ and $(x, y) \in L_{u}$. Then $y^{-1} x \in U$. By definition of semifundamental system, we can find $W \in S$ such that $W y^{-1} x \subseteq U$. Let $(x, z) \in L_{w^{\circ}}(x, y)$. Then $(y, z) \in L_{W}$ which implies that $z^{-1} y \in W$. Therefore, $z^{-1} y y^{-1} x \in W y^{-1} x \subseteq U$. Thus, $z^{-1} x \in U$ or equivalently, $(x, z) \in L_{U}$.

Let $L_{u} \in L$ and $(x, y) \in L_{y}$. Then $y^{-1} x \in U$. By definition of semifundamental system, we can find $W \in S$ such that $W Y^{-1} x \leq U$. Also, there exists $V \in S$ such that $y^{-1} x V\left(y^{-1} x\right)^{-1} \subseteq W$. So then $y^{-1} x V \subseteq W y^{-1} x \subseteq U$. Let $(z, y) \in(x, y) \circ L_{v}$. Then $(z, x) \in L_{v}$ which implies that $x^{-1} z \in V$. Therefore, $y^{-1} x^{-1} z \in y^{-1} x V \subseteq U$. Thus, $y^{-1} z \in U$, or equivalent $1 y,(z, y) \in L_{U}$.

To show that this left point semiuniformity generates the topology $\tau$, let $U \in \mathbb{S}$ and $x \in \mathbb{G}$. Then $L_{U}[x]=\left\{y \mid(x, y) \in L_{U}\right\}=\{y \mid x \in Y U\}=$ $\{\mathrm{Y} \mid \mathrm{Y} \in \mathrm{xU}\}=\mathrm{xU} . \bullet$

THEOREM 9. Suppose that $\mathbf{S}$ is a semifundamental system for a group $(\mathbb{G}, \cdot, \tau)$. If the left or right point semiuniformity is a uniformity, then $\mathbf{s}$ is a fundamental system.

PROOF. Assuming that $S$ generates a base for the left uniformity $L$, then picking $U \in S$ implies that $L_{U} \in L$ and so, by definition of $L, \exists V \in S$ such that $L_{v} \circ L_{v} \leq L_{v}$. If $x \in V \cdot V$ then $x=a \cdot b$ where $a \in V$ and $b \in V$. clearly, $x \in a \cdot V$ and $a \in e \cdot V=V$. Therefore, $(x, a) \in L_{v}$ and $(a, e) \in L_{v}$. Combining the above yields $(x, e) \in L_{v} \circ L_{v} \leq L_{U}$. Thus, $x \in e \cdot U=U$.

\section{REFERENCES}

1. WEIL, A. "Sur les espaces a structure uniforme et sur la topologie generale," Actualites Scientific and Industr., no. 551, (1937).

2. PAGE, W. Topological Uniform Structures, Wiley-Interscience Publishers, 1978 .

3. CLAY, E., CLARK, B., SCHNEIDER, V. "Semicontinuous Groups and Separation Properties," Internat. J. Math \& Math. Sci., vol. 15 no. 3 (1992), 621-623.

4. BOURBAKI, N. General Topology, parts 1 and 2, Addison-Wesley publishing Company, 1966.

5. FUCHS, L. Infinite Abelian Groups, Vol. 1, Academic Press, 1970 . 


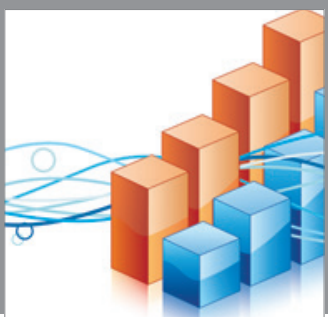

Advances in

Operations Research

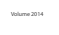

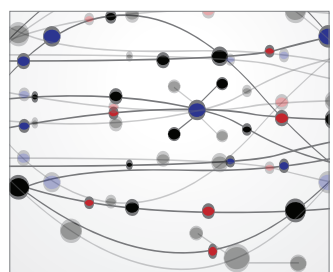

\section{The Scientific} World Journal
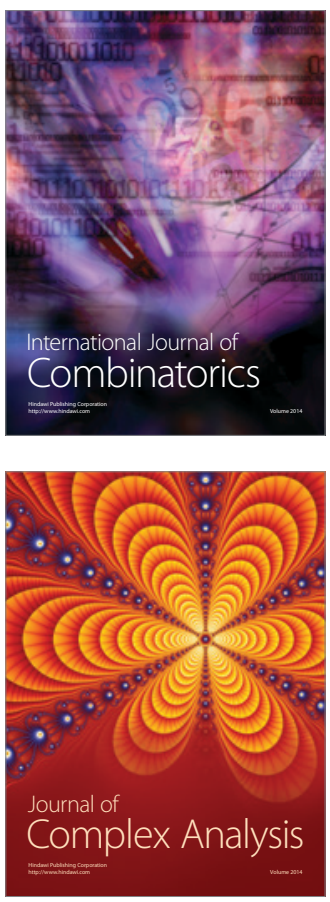

International Journal of

Mathematics and

Mathematical

Sciences
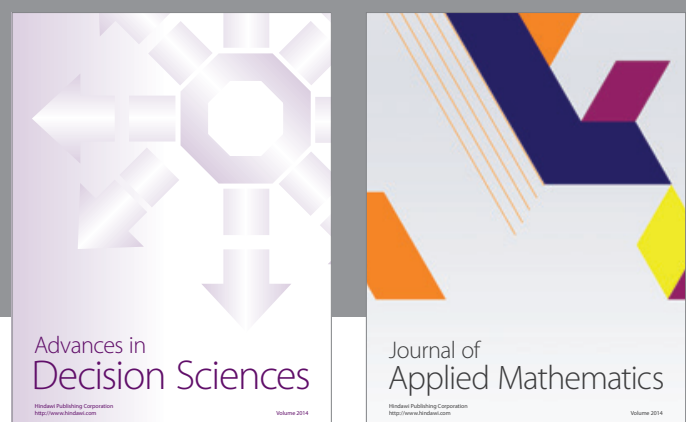

Journal of

Applied Mathematics
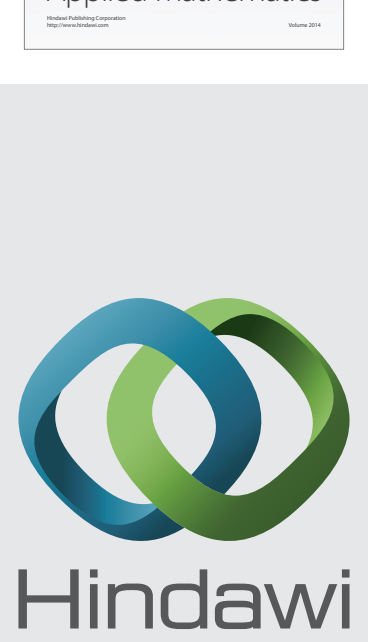

Submit your manuscripts at http://www.hindawi.com
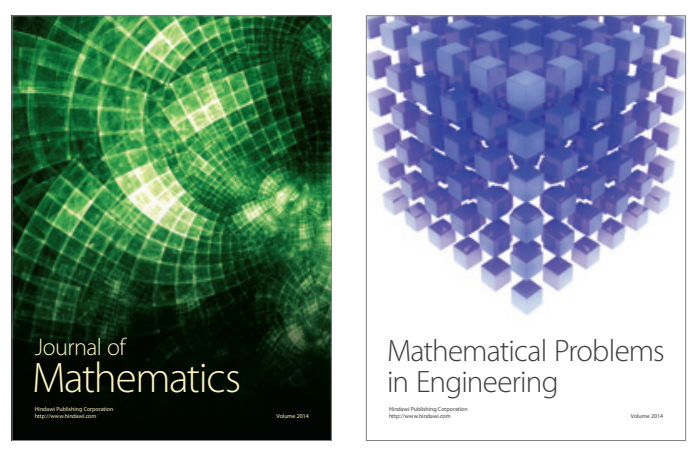

Mathematical Problems in Engineering
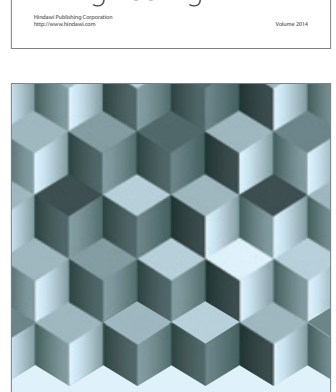

Journal of

Function Spaces
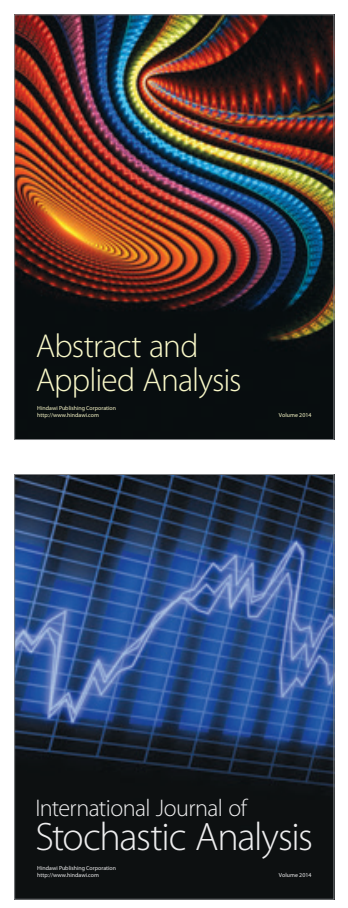

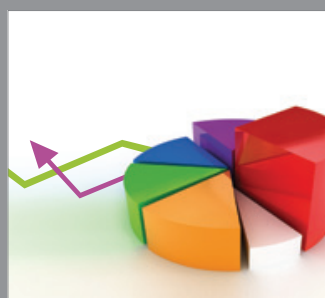

ournal of

Probability and Statistics

Promensencen
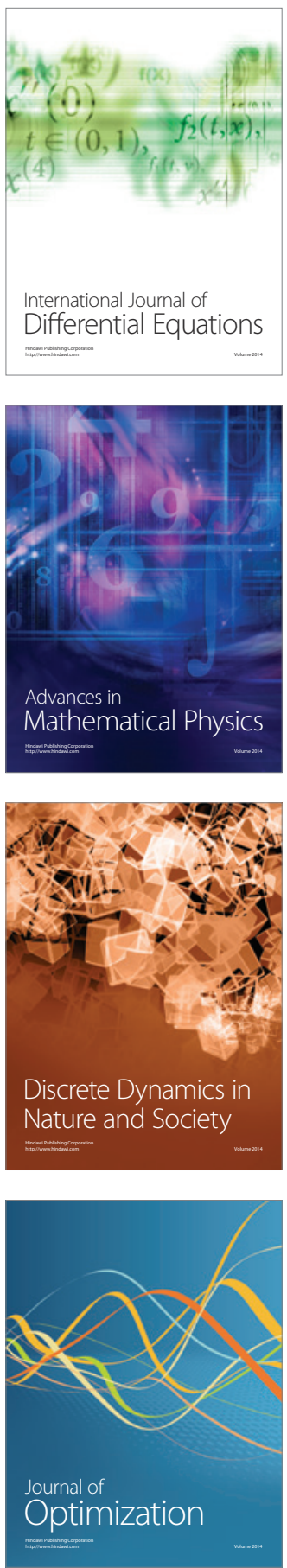